\title{
Correction: Assessment of the Effectiveness of Identity-Based Public Health Announcements in Increasing the Likelihood of Complying With COVID-19 Guidelines: Randomized Controlled Cross-sectional Web-Based Study
}

Alexander S Dennis ${ }^{1}$, BA; Patricia L Moravec ${ }^{2}$, BSc, MSc, PhD; Antino Kim³ ${ }^{3}$ BS, MS, MSBA, PhD; Alan R Dennis ${ }^{3}$, BCS, MBA, PhD

${ }^{1}$ Smith School of Business, University of Maryland, College Park, MD, United States

${ }^{2}$ McCombs School of Business, University of Texas at Austin, Austin, TX, United States

${ }^{3}$ Kelley School of Business, Indiana University, Bloomington, IN, United States

Corresponding Author:

Alan R Dennis, BCS, MBA, PhD

Kelley School of Business

Indiana University

1309 E 10th St

Bloomington, IN, 47405

United States

Phone: 18128552691

Email: ardennis@indiana.edu

\section{Related Article:}

Correction of: https://www.jmir.org/2021/4/e25762

(JMIR Public Health Surveill 2021;7(4):e29603) doi: 10.2196/29603

In "Assessment of the Effectiveness of Identity-Based Public Health Announcements in Increasing the Likelihood of Complying With COVID-19 Guidelines: Randomized Controlled Cross-sectional Web-Based Study" (JMIR Public Health Surveill 2021;7(4):e25762) the authors noted one error.

In the originally published manuscript, an Editorial Notice regarding the study's retrospective registration contained an incorrect quotation from the authors.

This randomized study was only retrospectively registered, explained by the authors with "this is a replication study". The editor granted an exception from ICMJE rules mandating prospective registration of randomized trials because the risk of bias appears low and the study was considered formative, guiding the development of the application, or specific requirements regarding preregestration. However, readers are advised to carefully assess the validity of any potential explicit or implicit claims related to primary outcomes or effectiveness, as retrospective registration does not prevent authors from changing

their outcome measures retrospectively.

The corrected version of this notice reads as follows:

This randomized study was only retrospectively registered as, according to the authors, their field has not adopted pre-registration as convention. The editor granted an exception from ICMJE rules mandating prospective registration of randomized trials because the risk of bias appears low. However, readers are advised to carefully assess the validity of any potential explicit or implicit claims related to primary outcomes or effectiveness, as retrospective registration does not prevent authors from changing their outcome measures retrospectively.

The correction will appear in the online version of the paper on the JMIR Publications website on April 15, 2021, together with the publication of this correction notice. Because this was made after submission to PubMed, PubMed Central, and other full-text repositories, the corrected article has also been resubmitted to those repositories. 
This is a non-peer-reviewed article. Submitted 13.04.21; accepted 13.04.21; published 15.04.21.

Please cite as:

Dennis AS, Moravec PL, Kim A, Dennis AR

Correction: Assessment of the Effectiveness of Identity-Based Public Health Announcements in Increasing the Likelihood of Complying

With COVID-19 Guidelines: Randomized Controlled Cross-sectional Web-Based Study

JMIR Public Health Surveill 2021;7(4):e29603

URL: https://publichealth.jmir.org/2021/4/e29603

doi: $10.2196 / 29603$

PMID:

(C)Alexander S Dennis, Patricia L Moravec, Antino Kim, Alan R Dennis. Originally published in JMIR Public Health and Surveillance (http://publichealth.jmir.org), 15.04.2021. This is an open-access article distributed under the terms of the Creative Commons Attribution License (https://creativecommons.org/licenses/by/4.0/), which permits unrestricted use, distribution, and reproduction in any medium, provided the original work, first published in JMIR Public Health and Surveillance, is properly cited. The complete bibliographic information, a link to the original publication on http://publichealth.jmir.org, as well as this copyright and license information must be included. 